\title{
New developments of multireflection grazing incidence diffraction
}

\author{
Marianna Marciszko ${ }^{1, a}$, Andrzej Baczmański ${ }^{1, b^{*}}$, Mirosław Wróbel ${ }^{1, \mathrm{c}}$, \\ Wilfrid Seiler ${ }^{2, d}$, Chedly Braham ${ }^{2, e}$ and Krzysztof Wierzbanowski ${ }^{1, f}$ \\ ${ }^{1}$ AGH University of Science and Technology, WFIS and WIMIP, al. Mickiewicza 30, 30-059 \\ Kraków, Poland \\ ${ }^{2}$ Arts et Métiers-ParisTech, PIMM, CNRS UMR 8006, 151 Bd de l'Hôpital, 75013 Paris, France

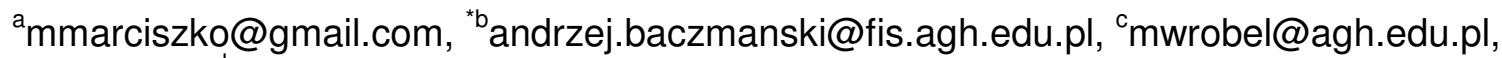 \\ wilfrid.seiler@paris.ensam.fr, ${ }^{\mathrm{e}}$ chedly.braham@paris.ensam.fr, \\ krzysztof.wierzbanowski@fis.agh.edu.pl
}

Keywords: grazing incidence, stacking faults, hexagonal structure, residual stresses

\begin{abstract}
The multireflection grazing incident X-ray diffraction (MGIXD) is used to determine a stress gradient in thin surface layers (about 1-20 $\mu \mathrm{m}$ for metals). In this work two theoretical developments of this method are presented. The first procedure enables determination of c/a parameter in hexagonal polycrystalline materials exhibiting residual stresses. In the second method, the influence of stacking faults on the experimental data is considered. The results of both procedures were verified using X-rays diffraction.
\end{abstract}

\section{Introduction}

Multireflection grazing incidence X-ray diffraction (MGIXD) is a non-destructive method which allows performing an analysis of the heterogeneous stress field for different volumes below the surface of the sample. The penetration depth of X-ray radiation is well defined and does not change during experiment in a wide $2 \theta$ range for a given incidence angle $\alpha[1,2,3]$. What is more the penetration depth can be changed by appropriate selection of $\alpha$ angle or/and by using different type of radiation which allows to investigate stress on different depths below sample surface. This gives a possibility to investigate materials with a stress gradient.

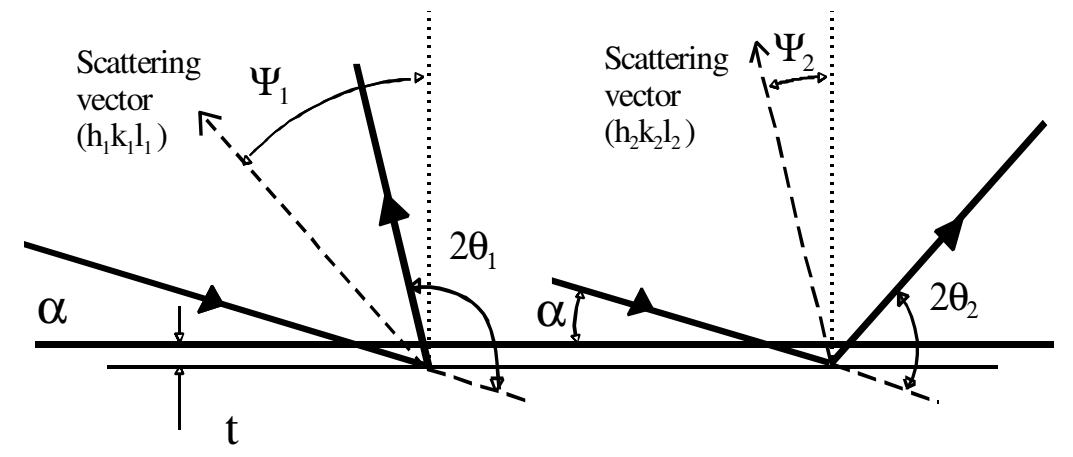

Fig.1. Geometry of MGIXD method. The incidence angle $\alpha$ is fixed during measurement while the orientation of the scattering vector is characterised by the angle $\psi_{\{h k l\}=}=\theta_{\{h k l\}}-\alpha$.

The MGIXD method allows determining macrostresses from measurements of different reflections which are more representative for the sample in comparison with single reflection methods, because strains in different crystallographic direction are simultaneously used in analysis. The MGIXD geometry is characterized by a small and constant incidence angle $\alpha$ (Fig.1) and by 
different orientations of the scattering vector (variable $2 \theta_{\{h k l\}}$ angle for a constant wavelength) given by the equation:

$$
\psi_{\{h k l\}=}=\theta_{\{h k l\}}-\alpha .
$$

Using fitting procedure stresses can be determined from the interplanar spacings $\langle d(\phi, \psi)\rangle_{\{h k l\}}$ measured in direction of the scattering vector for different $\psi_{\{h k l\}}$ (and consequently different $h k l$ reflections) and for constant $\alpha$ angle. In the case of multi-reflection method instead of $\left\langle d(\phi, \psi)_{\{h k l\}}\right.$, the so called equivalent lattice parameters $\left\langle a(\phi, \psi\rangle_{\{h k l\}}\right.$ are expressed by the macrostresses $\sigma_{i j}^{I}$ and strain free lattice constant $a_{0}$, using the X-ray stress factors $F_{i j}(h k l, \phi, \psi)$ [4]:

$$
<a(\phi, \psi\rangle_{\{h k l\}}=\left[F_{i j}(h k l, \phi, \psi) \sigma_{i j}^{I}\right] a_{0}+a_{0}
$$

where for cubic crystal structure: $\left\langle a(\phi, \psi)_{\{h k l\}}=\left\langle d(\phi, \psi)_{\{h k l\}} \sqrt{h^{2}+k^{2}+l^{2}}\right.\right.$

or for hexagonal structure: $\left\langle a(\phi, \psi)_{\{h k l\}}=<d(\phi, \psi)_{\{h k l\}}\left\{\left[\frac{4}{3}\left(h^{2}+h k+k^{2}\right)\right]+\frac{l^{2}}{(c / a)^{2}}\right\}^{-1 / 2}\right.$.

In the measurements the azimuth angle $\phi$ can be chosen arbitrary, while $\psi$ depends on the diffraction angle for given reflection $h k l$.

The penetration depth $\tau$ in MGIXD method is given by the well-known equation [5]:

$$
\tau=\left(\frac{\mu}{\sin \alpha}+\frac{\mu}{\sin \left(2 \theta_{\{h k l\}}-\alpha\right)}\right)^{-1}
$$

\section{Determination of c/a lattice parameter using MGIXD method}

In the case of cubic crystal structure the experimental $\langle a(\phi, \psi)\rangle_{\{h k l\}}$ lattice parameters are calculated directly from measured $\langle d(\phi, \psi)\rangle_{\{h k l\}}$ spacings (Eq. 2a). Subsequently, the $\sigma_{i j}^{I}$ and $a_{0}$ fitting parameters can be found by adjusting the $\left\langle a(\phi, \psi)_{\{h k l\}}\right.$ values obtained from Eq. 2 to the measured ones (Eq. 2a). However for hexagonal structure the value of $c / a$ parameter must be known a priori to calculate the experimental $\left\langle a(\phi, \psi\rangle_{\{h k l\}}\right.$ from Eq. $2 \mathrm{~b}$. To solve this problem the iteration method can be applied. In the first step the theoretical value of $c / a$ is inserted in Eq. $2 b$ and the least square method is used to find out $\sigma_{i j}^{I}$ and $a_{0}$ from Eq. 2. The result of the first adjustment is usually poor because the experimental $\langle a(\phi, \psi)\rangle_{\{h k l\}}$ are not correctly calculated using approximate value of $c / a$. Consequently the experimental $\langle a(\phi, \psi)\rangle_{\{h k l\}}$ do not agree with those obtained from Eq. 2 for optimized $\sigma_{i j}^{I}$ and $a_{0}$ fitting parameters. Therefore a procedure must be developed in order to correct the value of $c / a$ for the studied material, taking into account the macrostresses present in the sample. In this aim Eq. $2 \mathrm{~b}$ can be rewritten in the following form:

$$
y=p x
$$

where: $y=\left[\frac{\langle a(\phi, \psi)\rangle_{\{h k l\}}}{\langle d(\phi, \psi)\rangle_{\{h k l\}}}\right]^{2}-\left[\frac{4}{3}\left(h^{2}+h k+k^{2}\right)\right], \quad x=l^{2} \quad$ and $p=\frac{1}{(c / a)^{2}}$. 
The above linear vs. $l^{2}$ equation allows us to determine $p$ and consequently $c / a$ parameter using simple linear regression method. In calculations the measured $\langle d(\phi, \psi)\rangle_{\{h k l\}}$ spacings and values of $\langle a(\phi, \psi)\rangle_{\{h k l\}}$ calculated from Eq. 2 (for $\sigma_{i j}^{J}$ and $a_{0}$ optimized in the first step for approximate value of $c / a$ ) are substituted. It should be stated that the so obtained $c / a$ parameter is still approximate, but it can be applied in the second step of iteration to calculate $\left\langle a(\phi, \psi)_{\langle\{k l\}}\right.$ used in the least square procedure. As the result the new values of $\sigma_{i j}^{J}$ and $a_{0}$ are determined. It will be shown that two iteratively applied simple fitting procedures lead to convergence allowing determination of macrostresses $\sigma_{i j}^{J}$, strain free lattice parameter $a_{0}$ and moreover more accurate value of $c / a$. Finally, if the self-consistent iterative calculations are convergent a very good agreement between calculated values of $\left\langle a(\phi, \psi)_{\{h k l\}}\right.$ (obtained from Eq. 2) and experimental ones (determined from Eq. 2 b) can be reached.

\section{Influence of stacking faults on stress analysis}

Not only residual stresses are the reason of diffraction peaks shift with respect to the position corresponding to the perfect lattice but also stacking faults may be the cause [6]. One of the first works concerning this effect was done by Paterson [7], Warren et al. [8] and Wagner [9]. Wagner and Velterop et al. showed [10] that stacking faults can significantly change the position of the diffraction lines. This is especially important for the fcc crystals having low stacking fault energy (e.g. austenitic steels). In this case the magnitude of the displacement depends on the probability of finding the stacking fault and on the reflection $h k l$ used in the experiment [11]. In the absence of the second order incompatibility stresses it can be written [8]:

$<a(\phi, \psi)_{\{h k l\}}=\left[F_{i j}(h k l, \phi, \psi, f) \sigma_{i j}^{I}+\rho G(h k l)\right] a_{0}+a_{0}$
with $G(h k l) \cong-\frac{\sqrt{3}}{4 \pi m} \sum_{m} \frac{s L_{0}}{h^{2}+k^{2}+l^{2}} ; L_{0}=h+k+l ; s=\left\{\begin{array}{l}-1 \text { for } L_{0}=3 M-1 \\ 0 \text { for } L_{0}=3 M \\ +1 \text { for } L_{0}=3 M+1\end{array}\right.$ and $M \in \mathbb{Z}$,

where $G(h k l)$ is the coefficient reflecting relative changes of the determined interplanar spacings caused by stacking faults, the sum is calculated over all symmetrically equivalent $\{h k l\}$ planes, $m$ is the plane multiplicity, $\rho$ is the probability of finding the deformation stacking fault between neighboring planes $\{111\}$.

Both the macrostress and stacking faults cause the nonlinearities of the $\langle a(\phi, \psi)\rangle_{\{h k l\}}$ vs. $\sin ^{2} \psi_{\{h k l\}}$ plots. Macrostresses influence the slope as well as the nonlinearites of the curve. In contrast the stacking faults increase only the nonlinearities of these plots. This fact allows to separate the effect originated from the stresses from the one connected with the stacking faults and perform the calculation of stresses values and the probability of stacking faults in a polycrystal. The idea of fitting is similar to that used in determination of the second order stresses, when $q$ scaling factor was used in Eq. 5 as additional adjusting parameter [12]. The value of $\rho$ is varied in fitting procedure in order to receive the best agreement of theoretic and experimental results. The optimized $\rho$ parameter has meaning of probability of finding stacking fault between neighboring planes $\{111\}$.

\section{Experimental results}

In order to verify the proposed iteration method of c/a parameter determination a polished $\mathrm{Ti}$ (grade 2) sample was investigated. Measurements were performed in two directions (i.e. for $\phi=0^{\circ}$ 
and $\phi=90^{\circ}$ ) and for $\alpha=5^{\circ}$ incidence angle, with $\mathrm{Cu} \mathrm{X}$-ray tube and Göbel mirror in the incidence beam optics. The PANalytical - X'Pert MRD diffractometer was used. The influence of the stacking fault presence on stress analysis was investigated on austenitic stainless steel sample. The composition of the examined samples is given in Table 1.

Table 1. Composition of the materials used in thesis (wt.\%).

\begin{tabular}{|c|cccccccccc|}
\hline $\mathrm{Ti}$ & $\mathrm{Ti}$ & $\mathrm{O}$ & $\mathrm{Fe}$ & $\mathrm{Ni}$ & $\mathrm{C}$ & $\mathrm{N}$ & & & & \\
grade 2 & bal. & 0.131 & 0.109 & 0.020 & 0.010 & 0.010 & & & & \\
\hline Austenite & $\mathrm{Fe}$ & $\mathrm{Cr}$ & $\mathrm{Ni}$ & $\mathrm{Mo}$ & $\mathrm{Mn}$ & $\mathrm{Cu}$ & $\mathrm{Si}$ & $\mathrm{P}$ & $\mathrm{S}$ & $\mathrm{C}$ \\
AISI316L & bal. & 17.24 & 11.14 & 1.96 & 1.67 & 0.35 & 0.056 & 0.04 & 0.04 & 0.02 \\
\hline
\end{tabular}

The stress calculation was performed with the XSF (X-ray stress factors [6]) calculated using Kröner model and from single crystal elastic constants given in Table 2. The values of XSF were positively verified measuring lattice strains for tensile loads applied to the sample $[13,14,15]$.

Table 2. Single crystal elastic constants $\left(\mathrm{C}_{\mathrm{ij}}\right)$ and for studied materials [16].

\begin{tabular}{|c|c|c|c|c|c|c|}
\hline Material & $\mathrm{C}_{11}$ & $\mathrm{C}_{12}$ & $\mathrm{C}_{13}$ & $\mathrm{C}_{33}$ & $\mathrm{C}_{44}$ & $\mathrm{C}_{66}$ \\
\hline $\mathrm{Ti}$ & 162 & 92 & 69 & 180 & 47 & 35 \\
\hline $\begin{array}{l}\text { Austenite } \\
\text { AISI316L }\end{array}$ & 197 & 122 & 122 & 197 & 124 & 124 \\
\hline
\end{tabular}

At first the calculation of the stresses in polished Ti (grade 2) was performed using assumed values of $c / a$ parameter indicated in Fig. 2. In this case the value of $c / a$ was not varied during data treatment. It can be noticed that the experimental points are spread far from the lines obtained by fitting Eq. 2.

Next, the self-consistent procedure was used and the value $c / a$ was also adjusted. The resulting $\langle a(\phi, \psi)\rangle_{\{h k l\}}$ vs. $\sin ^{2} \psi$ plots exhibit significantly better agreement between theoretical and experimental points (Fig. 2 c). The values of $c / a$ parameter and goodness of fitting $\chi^{2}$ determined using the presented above procedure are given in these figures. Goodness of fitting was calculated from:

$$
\chi^{2}=\frac{1}{N-M} \sum_{n=1}^{N}\left(\frac{<a\left(\phi_{n}, \psi_{n}\right)>_{\{h k l\}}^{\exp }-<a\left(\phi_{n}, \psi_{n}\right)>_{\{h k l\}}^{c a l}}{\delta\left(<a\left(\phi_{n}, \psi_{n}\right)>_{\{h k l\}}\right)}\right)^{2}
$$

where $<a\left(\phi_{n}, \psi_{n}>_{\{h k l\}}^{e x p}\right.$ and $\left\langle a\left(\phi_{n}, \psi_{n}>_{\{h k l\}}^{c a l}\right.\right.$ are experimental and calculated equivalent lattice parameters determined using $h k l$ reflections, $\delta_{n}\left(\left\langle a(\phi, \psi)_{\{h k l\}}\right)\right.$ is an experimental uncertainty of the spacing $<a\left(\phi_{n}, \psi_{n}>_{\{h k l\}}^{\text {exp }}\right.$ for the $n$-th measurement, $N$ and $M$ are the numbers of measurement points and fitting parameters, respectively.

In Fig. 2 it can be seen that value of $\chi^{2}$ decreases significantly when experimental points approach the theoretical curves. The results of self-consistent fitting for mechanically polished $\mathrm{Ti}$ sample are gathered in Table 3. 


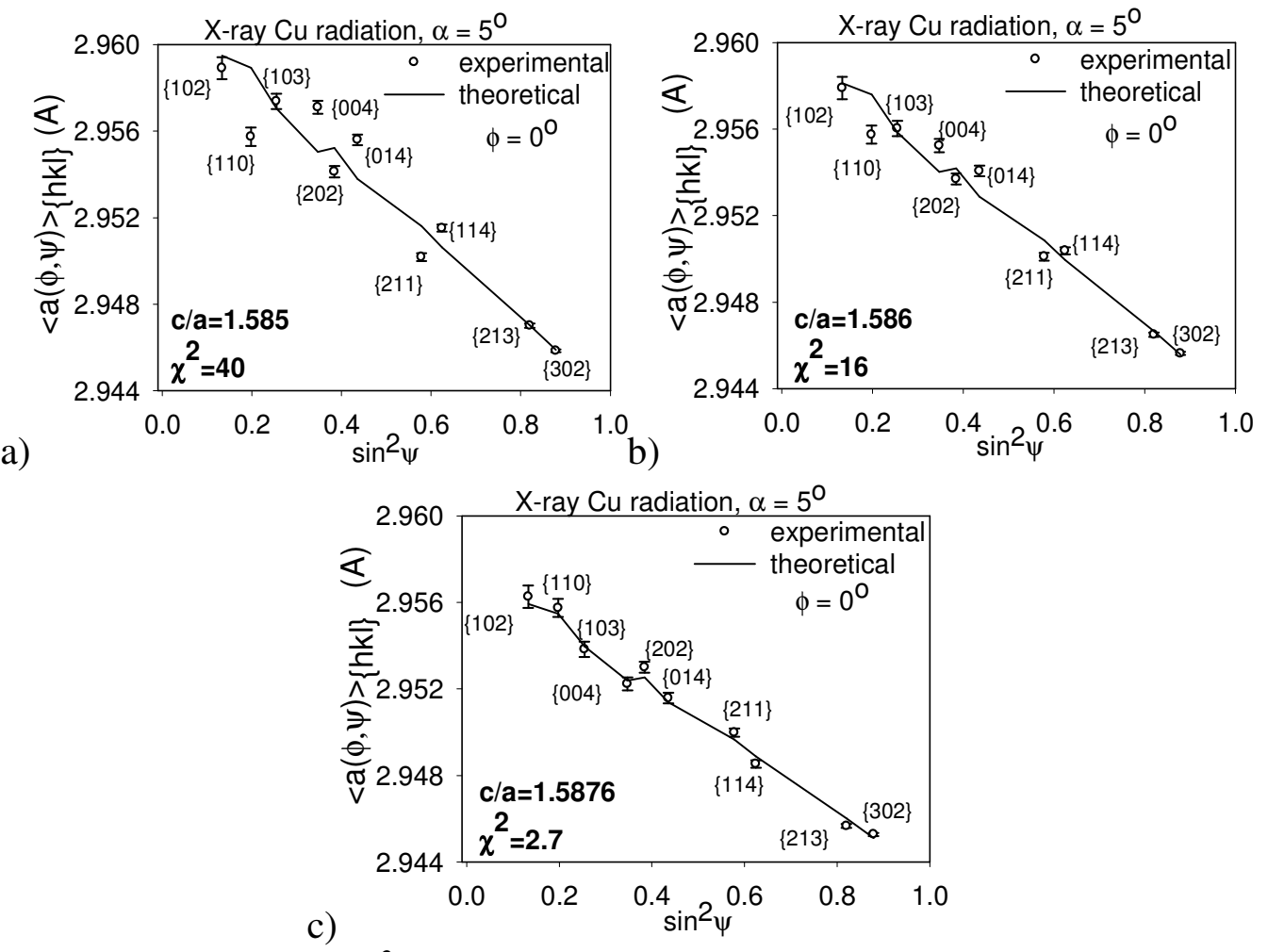

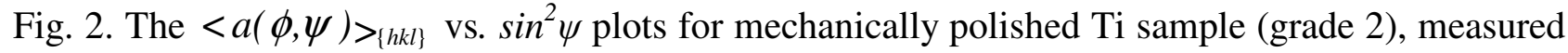
with $\alpha=5^{\circ}$. In figures (a) and (b) the theoretical plots were fitted to experimental points determined with assumed $c / a$ values, while in the case of figure (c) the $c / a$ parameter was adjusted. Uncertainty of peak position $\delta(2 \theta)=0.01^{\circ}$ was assumed.

Table 3. The results of self-consistent fitting for mechanically polished Ti sample, measured with $\alpha$ $=5^{\circ}$. The following values are presented: stresses in two directions, $\chi^{2}$ - goodness of fitting and lattice parameters.

\begin{tabular}{|c|c|c|c|c|c|}
\hline$\sigma_{11}(\mathrm{MPa})$ & $\sigma_{22}(\mathrm{MPa})$ & $\mathrm{a}_{0}(\AA)$ & $\mathrm{c}_{0}(\AA)$ & $\mathrm{c} / \mathrm{a}$ & $\chi^{2}$ \\
\hline$-392 \pm 10$ & $-367 \pm 10$ & $2.9510 \pm 0.0001$ & $4.6851 \pm 0.0001$ & $1.5876 \pm 0.0001$ & 2.7 \\
\hline
\end{tabular}

It should be underlined that the obtained lattice parameters are very close to the accurate values for high purity Ti [17]: $a_{0}=2.95111 \pm 0.00006 \AA, c_{0}=4.68433 \pm 0.0001 \AA$ and $\mathrm{c} / \mathrm{a}=1.5873$. Finally it should be stated that the new methodology of experimental data treatment enables determination not only the strain free $a_{0}$ constant but also the $c / a$ parameter.

As it was mentioned the influence of the stacking faults on the stress analysis was investigated for austenitic stainless steel subjected to mechanical treatment of the surface. The presented methodology of stress and stacking faults analysis requires knowledge of XSF for anisotropic material (like austenite stainless steel). This is why different models for calculations of XSF were verified by measuring lattice strains during tensile test. It was found that the free-surface model [12] fits correctly to the experimental results $[13,14]$. Therefore this model was used to calculate XSF from single crystal elastic constants (Table 2), taking into account experimentally determined crystallographic texture.

Measurements for ground and mechanically polished austenitic samples were performed in two directions (i.e. for $\phi=0^{\circ}$ and $\phi=90^{\circ}$ ) and for $\alpha=10^{\circ}$ incidence angle, using the Fe X-ray tube (Seifert - PTS MZ VI) and Cu X-ray tube (PANalytical - X'Pert MRD), respectively. The $\sin ^{2} \psi$ plots for analyzed sample are presented on Fig. 3. Dashed line presents analysis when stacking faults were not taken into account while continuous line presents analysis when stacking faults were taken into account. The results of the data analysis are gathered and compared in Table 4. 

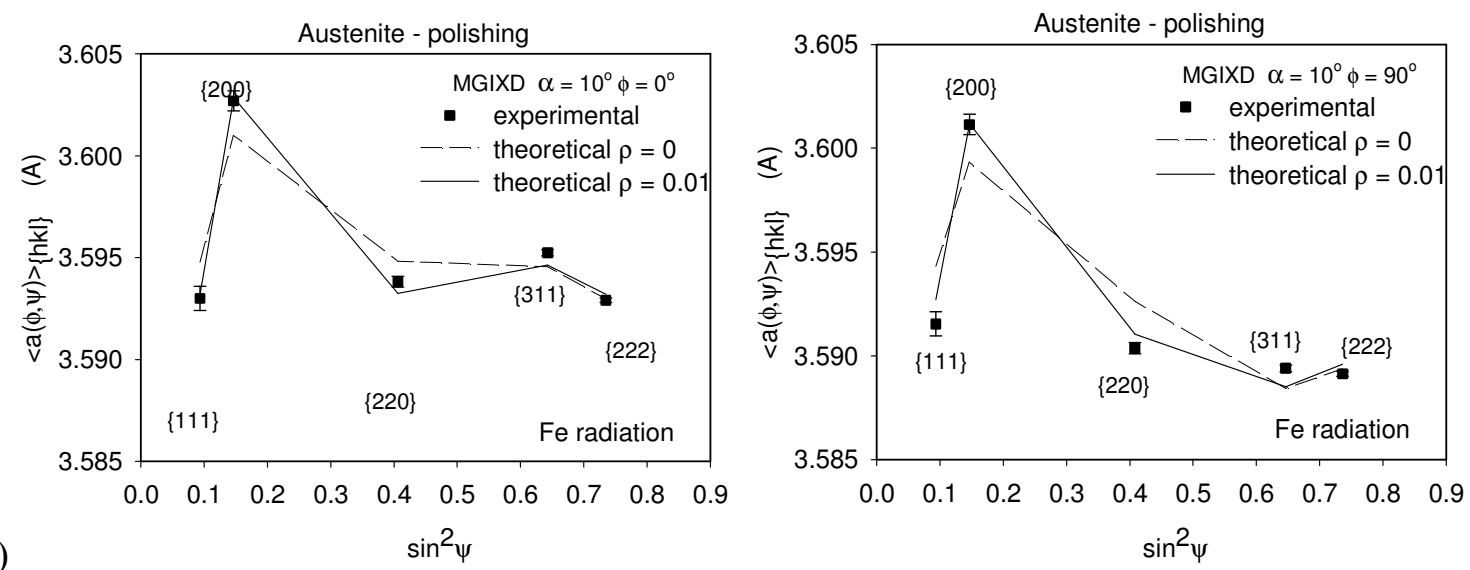

a)

$$
\sin ^{2} \psi
$$
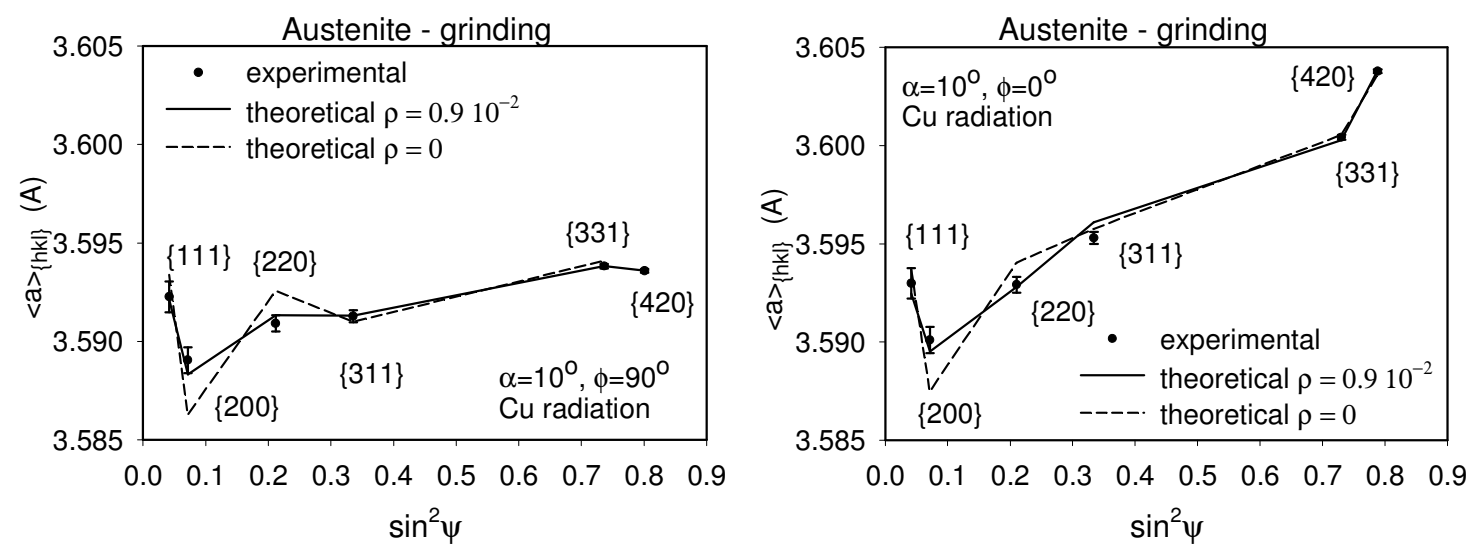

b)

Fig. 3. The $\langle a(\phi, \psi)\rangle_{\{h k l\}}$ lattice parameters fitted to the experimental points (assuming $\rho \neq 0$ continuous line or $\rho=0$ - dashed line) for the polished (a) and ground (b) austenitic steel - AISI 316L, XSF were calculated with free surface model [12].

Table 4. The results of self-consistent fitting for mechanically polished austenitic stainless steel sample, measured with $\alpha=10^{\circ}$. The following values are presented: stresses in two directions, $\chi^{2}$ goodness of fitting and lattice parameters.

\begin{tabular}{|c|c|c|c|c|c|}
\hline Sample & $\rho(\%)$ & $\sigma_{11}(\mathrm{MPa})$ & $\sigma_{22}(\mathrm{MPa})$ & $\mathrm{a}_{0}(\AA)$ & $\chi^{2}$ \\
\hline polished & 0 & $-215 \pm 78$ & $-533 \pm 74$ & $3.5934 \pm 0.0004$ & 29 \\
\cline { 2 - 6 } AISI 316L & $1.0 \pm 0.3$ & $-209 \pm 53$ & $-528 \pm 51$ & $3.5930 \pm 0.0003$ & 13 \\
\hline ground & 0 & $603 \pm 32$ & $143 \pm 31$ & $3.5950 \pm 0.0003$ & 8.4 \\
\cline { 2 - 6 } AISI 316L & $0.9 \pm 0.1$ & $608 \pm 13$ & $149 \pm 13$ & $3.5949 \pm 0.0001$ & 1.5 \\
\hline
\end{tabular}

For austenite stainless steel having low energy of stacking faults it would appear likely that taking into account the presence of stacking faults in stress analysis can be beneficial. Admittedly the stacking fault effect improves the fit of the theoretical curve (calculated from the chosen grain interaction model) to experimental points. 


\section{Summary}

The method of $c / a$ determination was proposed and tested for hexagonal samples with residual stresses (polished $\mathrm{Ti}$ - grade 2). It was shown that for the material having low elastic anisotropy of the stresses, strain free parameter $a_{0}$ as well as $c / a$ value can be determined using the self-consistent iteration method presented in this work. Significantly better fitting of the theoretical values to experimental ones was obtained when $c / a$ was adjusted.

Probability of finding stacking fault $\rho$ was determined for polished austenitic sample (alloy having low stacking fault energy). Reasonable values of $\rho$ but with very large uncertainty were determined in the case of compressive stress in the polished sample and tensile stresses in ground sample.

Acknowledgements. This work was financed by the grants from Polish National Scientific Center (NCN): UMO-2011/03/N/ST8/04058 and DEC-2011/01/B/ST8/07394. The support of the Polish Ministry of Science and Higher Education (MNiSW) is also acknowledged.

\section{References}

[1] Ch. Genzel, Formalism for the evaluation of strongly non-linear surface stress fields by X-ray diffraction performed in the scattering vector mode, Phys. Status Solidi A, 146 (1994) 629.

[2] A. Kumar, U. Welzel and E. J. Mittemeijer, A method for the non-destructive analysis of gradients of mechanical stresses by X-ray diffraction measurements at fixed penetration/information depths, J. Appl. Cryst. 39 (2006) 633.

[3] P. Predecki, B. Ballard and X. Zhu, Proposed methods for depth profiling of residual stresses using grazing incidence X-ray diffraction, Adv. X-ray Anal. 3 (1993) 237.

[4] Skrzypek S.J., Baczmański A., Ratuszek W., Kusior E., New approach to stress analysis based on a grazing-incidence X-ray diffraction, J. Appl. Cryst., 34 (2001) 427.

[5] François M., Unified description for the geometry of X-ray stress analysis, proposal for a consistent approach, J. of Appl. Cryst. 41 (2008) 44.

[6] Hauk V., Structural and Residual Stress Analysis by Nondestructive Methods, Elsevier, Amsterdam (1997)

[7] Paterson M.S., X-ray diffraction by face-centered cubic crystals with deformation faults, J.Appl.Phys., 23 (1952) 805.

[8] Warren B.E, Warekois E.P., Stacking faults in cold worked alpha-brass, Acta Met. 3 (1955) 473.

[9] Wagner C.N.J., Proc. Of Symp., Local Atomic Arrangements studied by X-ray Diffraction, Metall. Soc. Conferences 36 (1966) 219.

[10] Velterop L., Delhez R., de Keijser Th.H., Mittemeijer E.J., Reefman D., X-ray diffraction analysis of stacking and twin faults in fcc metals: a revision and allowance for texture and nonuniform fault probabilities, J. Appl. Cryst. 33 (2000) 296.

[11]Jegou S., Christiansen T.L., Klaus M., Genzel Ch., Somers M.A.J., Determination of composition, residual stress and stacking fault depth profiles in expanded austenite with energydispersive diffraction, Thin Solid Films 530 (2013) 71.

[12] Baczmański A., Lipinski P., Tidu A., Wierzbanowski K., Pathiraj B., Quantitive estimation of incompatibility stresses and elastic energy stored in ferritic steel, J. Appl. Cryst. 41 (2008) 854.

[13] Marciszko, M., Diffraction study of mechanical properties and residual stresses resulting from surface processing of polycrystalline materials, PhD Thesis, AGH-Krakow and ENSAM Paris, 2013.

[14] Marciszko M., Baczmański A., Wroński S., Seiler W., Braham C., Wróbel M., Anisotropy of $\mathrm{X}$-ray stress factors used in the grazing incident measurement, to be presented on ECRS 9, Troyes, 2014. 
[15] U. Welzel, J. Ligot, P. Lamparter, A. C. Vermeulenb and E. J. Mittemeijer, (2005). J. Appl. Cryst. 38, 1-29.

[16] Simoms G., Wang H., Single Crystal Elastic Constants and Calculated Aggregate Properties: A handbook, second edition, The M.I.T. Press, Cambridge, Massachusetts and London, 1971.

[17] Wood R.M., The Lattice Constants of High Purity Alpha Titanium, Proc. Phys. Soc. 80 (1962) 783. 